\title{
Blended General Linear Methods based on Generalized BDF
}

\author{
Luigi Brugnano* and Cecilia Magherini ${ }^{\dagger}$ \\ *Dipartimento di Matematica “U. Dini”, Viale Morgagni 67/A, 50134 Firenze (Italy) \\ luigi.brugnano@unifi.it \\ ${ }^{\dagger}$ Dipartimento di Matematica Applicata “U. Dini”, Via Buonarroti 1/C , 56127 Pisa (Italy) \\ cecilia.magheriniedma.unipi.it
}

\begin{abstract}
General Linear Methods were introduced in order to encompass a large family of numerical methods for the solution of ODE-IVPs, ranging from LMF to RK formulae. In so doing, it is possible to obtain methods able to overcome typical drawbacks of the previous classes of methods. For example, stability limitations of LMF and order reduction for RK methods. Nevertheless, these goals are usually achieved at the price of a higher computational cost. Consequently, many efforts have been done in order to derive GLMs with particular features, to be exploited for their efficient implementation.

In recent years, the derivation of GLMs from particular Boundary Value Methods (BVMs), namely the family of Generalized BDF (GBDF), has been proposed for the numerical solution of stiff ODE-IVPs. Here, this approach is further developed in order to derive GLMs combining good stability and accuracy properties with the possibility of efficiently solving the generated discrete problems via the blended implementation of the methods.
\end{abstract}

Keywords: Numerical methods for ordinary differential equations, General Linear Methods, Boundary Value Methods (BVMs), Generalized Backward Differentiation Formulae (GBDF), Blended Implicit Methods, blended iteration.

PACS: 65L05,65L20,65H10,65Y20.

\section{INTRODUCTION}

General Linear Methods, in the form introduced by Burrage and Butcher [10], are numerical methods for solving ODEIVPs which, in order to advance the integration of one step, require some information from the previous step (external stages), along with some internal stages to be computed at the current step. Such methods are able to describe, as extreme cases, both RK methods and LMF (the latter, when used as Initial Value Methods (IVMs)) [11]. A GLM with $r$ external stages and $s$ internal stages, when applied with stepsize $h$ for the solution of the IVP

$$
y^{\prime}=f(t, y), \quad y\left(t_{0}\right)=y_{0} \in \mathbb{R}^{m},
$$

is usually described as

$$
Y^{[n]}=h\left(A \otimes I_{m}\right) F^{[n]}+\left(U \otimes I_{m}\right) \mathbf{y}^{[n-1]}, \quad \mathbf{y}^{[n]}=h\left(B \otimes I_{m}\right) F^{[n]}+\left(V \otimes I_{m}\right) \mathbf{y}^{[n-1]}, \quad n=1,2, \ldots,
$$

where: $A \in \mathbb{R}^{s \times s}, U \in \mathbb{R}^{s \times r}, B \in \mathbb{R}^{r \times s}, V \in \mathbb{R}^{r \times r} ; Y^{[n]}, F^{[n]} \in \mathbb{R}^{s m}$ are the vector of the internal stages and the corresponding values of the function $f$, respectively; $\mathbf{y}^{[n]} \in \mathbb{R}^{r m}$ is the vector of the external stages. Many papers have been devoted, across the years, to the construction and the analysis of GLMs (see, e.g., $[12,13,14,15,16,17$, $18,19,20])$. Clearly, the simpler the form of the matrix $A$, the more efficient the implementation of the corresponding GLM. In this setting, we shall consider implicit GLMs for which $A=B, U=V \in \mathbb{R}^{r \times r}$ and, therefore, $Y^{[n]}=\mathbf{y}^{[n]}$, i.e., the external and the internal stages coincide. Moreover, the order of accuracy of each entry of $\mathbf{y}^{[n]}$ is equal to $p$ (to be specified later), which is, therefore, the global order of the method. We mention that sometimes GLMs with approximations having the same order have been also called "peer methods" (see, e.g., [21]). The approach that we shall consider is the one introduced in [8, Chapter 11, Section 6] and is based on Boundary Value Methods (BVMs). In more details, the family of BVMs which we shall consider is that of Generalized Backward Differentiation Formulae (GBDF) [7], which is briefly sketched in the next section, along with their formulation as GLMs. For the efficient solution of the generated discrete problems, in the last section we shall consider the blended implementation of such methods. This implementation, introduced in [1] for block implicit methods (see also [2, 3, 4, 6, 8, 9]), naturally induces an efficient splitting procedure for the solution of the generated discrete problems. The linear analysis of convergence for this splitting is done according to [5]. 


\section{GENERALIZED BACKWARD DIFFERENTIATION FORMULAE (GBDF) AND GENERAL LINEAR METHODS}

Let us consider the particular class of $k$-step LMF having the polynomial $\sigma(z)$ in its simplest form,

$$
\sigma(z)=z^{j}
$$

where $j \in\{0,1, \ldots, k\}$, with the coefficients of the corresponding polynomial $\rho(z)=\sum_{i=0}^{k} \alpha_{i} z^{i}$ uniquely defined by imposing that the method has the maximum possible order $p=k$. When $j=k$, one obtains the well-known family of BDF which, however, provides 0 -stable methods only up to $k=6$ and $A$-stable methods up to $k=2$. Nevertheless, when

$$
j=v \equiv\lceil(k+2) / 2\rceil,
$$

and the formula is used as a BVM with $(v, k-v)$-boundary conditions, i.e., by fixing the first $v$ values and the final $k-v$ values of the discrete solution, then the methods turns out to be stable for all values of $k$ [7, 8]. By the way, when $k=1,2$, one obtains the usual first two BDF. In view of the implementation of GBDF as GLMs, one may assume to know the initial values of the discrete solution. On the other hand, the final $k-v$ values can be retrieved implicitly by using a suitable set of additional final methods [8], consisting of LMF having the following characteristic polynomials:

$$
\sigma(z)=z^{j}, \quad \rho(z)=\sum_{i=0}^{k} \alpha_{i}^{(j)} z^{i}, \quad j=v+1, \ldots, k,
$$

with the coefficients $\left\{\alpha_{i}^{(j)}\right\}$ uniquely defined by imposing the order $p=k$ conditions. If we assume, for sake of simplicity, that a constant stepsize $h$ is used, then by introducing the vectors

$$
\mathbf{y}_{\text {new }}=\left(y_{n+1}, \ldots, y_{n+r}\right)^{T}, \quad \mathbf{f}_{\text {new }}=\left(f_{n+1}, \ldots, f_{n+r}\right)^{T}, \quad \mathbf{y}_{\text {old }}=\left(y_{n-r+1}, \ldots, y_{n}\right)^{T},
$$

and the $r \times r$ matrices $A_{1}$ and $A_{2}$ such that

$$
\left[A_{1} \mid A_{2}\right]=\left(\begin{array}{cccc|cccc}
\alpha_{0} & \ldots & \ldots & \ldots & \ldots & \alpha_{k} & & \\
& \ddots & & & & & \ddots & \\
& & \alpha_{0} & \ldots & \ldots & \ldots & \ldots & \alpha_{k} \\
& \alpha_{0}^{(v+1)} & \ldots & \ldots & \ldots & \ldots & \alpha_{k}^{(v+1)} \\
& \vdots & & & & & \vdots \\
& & \alpha_{0}^{(k)} & \ldots & \ldots & \ldots & \ldots & \alpha_{k}^{(k)}
\end{array}\right),
$$

the discrete problem, at a given point $t_{n}=n h$, can be cast in matrix form as

$$
\left(A_{2} \otimes I_{m}\right) \mathbf{y}_{\text {new }}=h \mathbf{f}_{\text {new }}-\left(A_{1} \otimes I_{m}\right) \mathbf{y}_{\text {old }} .
$$

This corresponds to a GLM (2) with coinciding internal and external stages, and $A=A_{2}^{-1}, U=-A_{2}^{-1} A_{1}$ (indeed, matrix $A_{2}$ turns out to be nonsingular). Such methods were called Block BVMs with Memory $\left(\mathrm{B}_{2} \mathrm{VM}_{2} \mathrm{~s}\right)$ in [8]. In the GLM notation, the abscissae defining this GLM are $c_{i}=i, i=1,2, \ldots, r$. However, in order to guarantee the $L$-stability of the corresponding method, it is sometimes required to change them into the following set of abscissae (as suggested in [8]):

$$
c_{i}=i, \quad i=1, \ldots, \ell-1, \quad c_{\ell-1+j}=\ell-1+\sum_{m=1}^{j} \zeta^{m}, \quad j=1, \ldots, r-\ell+1, \quad \text { with } \quad \zeta \in(0,1] \quad \text { s.t. } \quad c_{r}=\ell .
$$

Clearly, when $\ell=r$ we have a uniform mesh, whereas for $\ell<r$ the last $r-\ell+1$ points are spaced with geometrically decreasing stepsizes. In such a case, the points corresponding to the abscissae $c_{1}, \ldots, c_{\ell-1}, c_{r}$ are equally spaced and are the ones needed for the subsequent integration step. This implies that corresponding $r-\ell$ zero columns must be appropriately inserted in matrix $A_{1}$ in (3). Consequently, such a GLM based on GBDF is uniquely determined by the triple of integers $(k, r, \ell)$ (clearly, the order of the corresponding method is $p=k$ ). By choosing appropriately the values of $(r, \ell)$, it is possible to guarantee the $L$-stability of such methods for all practical values of $k$. However, it turns out that, for each value of $k$, the couples $(r, \ell)$ are not unique. Consequently, an additional criterion will be considered, in the next section, in order to speed-up the iterative solution of the corresponding discrete problems, via the blended implementation of the methods. 


\section{BLENDED GENERAL LINEAR METHODS}

In the previous section, we devised a procedure for obtaining a whole class of implicit GLMs based on GBDF, containing $L$-stable methods of arbitrarily high order. We now consider the problem of efficiently solving the generated discrete problems which, at a given time step, assumes the form (see (2)), $\mathbf{y}-h\left(A \otimes I_{m}\right) \mathbf{f}=\eta$, where $\eta$ is a known vector and $A$ is a nonsingular matrix. In order to describe the blended implementation of such method (see, e.g., $[1,2,3,4,5,6])$, the given GLM is conveniently applied to the usual test equation, $y^{\prime}=\lambda y, \operatorname{Re}(\lambda)<0$, for which the discrete problem reduces to a linear system of dimension $r$ :

$$
(I-q A) \mathbf{y}=\eta, \quad q=h \lambda .
$$

Such linear system is clearly equivalent to

$$
\gamma\left(A^{-1}-q I\right) \mathbf{y}=\gamma A^{-1} \eta \equiv \eta_{1}
$$

with $\gamma>0$ a free parameter. By introducing the weight function $\theta(q)=(1-\gamma q)^{-1} I$, we obtain an equivalent discrete problem by combining, with weights $\theta(q)$ and $I-\theta(q)$, respectively, the linear systems (4)-(5):

$$
M(q) \mathbf{y} \equiv\left(\theta(q)(I-q A)+\gamma(I-\theta(q))\left(A^{-1}-q I\right)\right) \mathbf{y}=\theta(q) \eta+\gamma(I-\theta(q)) \eta_{1} \equiv \eta(q) .
$$

Equation (6) defines the blended GLM corresponding to the original GLM (4). In view of the fact that $M(q) \approx I$, for $q \approx 0$, and $M(q) \approx-\gamma q I$, for $|q| \gg 1$, the following blended iteration for solving the discrete problem (6) is naturally induced:

$$
N(q) \mathbf{y}^{(i+1)} \equiv(I-\gamma q I) \mathbf{y}^{(i+1)}=(N(q)-M(q)) \mathbf{y}^{(i)}+\eta(q), \quad i=0,1, \ldots
$$

By considering that $N(q)^{-1}=\theta(q)$, it is quite straightforward to realize that, in the case of problem (1), the corresponding blended iteration requires only the factorization of one matrix of the size of the continuous problem, since, in this case, $\theta=I \otimes\left(I_{m}-\gamma h J\right)$, with $J$ the Jacobian evaluated at the last known point. We observe that (see [5]) the iteration matrix corresponding to the blended iteration (7) turns out to be given by

$$
Z(q) \equiv I-N(q)^{-1} M(q)=\frac{q}{(1-\gamma q)^{2}} A^{-1}(A-\gamma I)^{2} .
$$

Such matrix is zero at $q=0$ and at $\infty$ and its spectral radius, $\rho(q)$, is such that

$$
\rho(q) \approx \tilde{\rho} q, \quad q \approx 0, \quad \rho(q) \approx \frac{\tilde{\rho}_{\infty}}{q}, \quad|q| \gg 1, \quad \rho^{*}=\max _{x>0} \rho(i x),
$$

where $\tilde{\rho}, \tilde{\rho}_{\infty}$, and $\rho^{*}$ are the nonstiff amplification factor, the stiff convergence factor, and the maximum amplification factor, respectively, which, according to [5], are evaluation parameters for the blended iteration (7). In particular, if $\rho^{*} \leq 1$, the iteration is $L$-convergent and, then, appropriate for $L$-stable methods [5]. Moreover, it turns out that

$$
\tilde{\rho}=\rho\left(A^{-1}(A-\gamma I)^{2}\right), \quad \tilde{\rho}_{\infty}=\gamma^{-2} \tilde{\rho}, \quad \rho^{*}=(2 \gamma)^{-1} \tilde{\rho},
$$

and the value of the free positive parameter $\gamma$ is chosen in order to minimize $\rho^{*}$. In Table 1 , the relevant figures are reported for selected GLMs based on GBDF, each characterized by the corresponding triple $(k, r, \ell)$. As it can be seen, the blended iteration corresponding to each method turns out to be $L$-convergent. Moreover, the value of the parameter $\gamma$ (with the only exception of that corresponding to $k=4$ ) turns out to coincide with the value, $\gamma^{*}=\min _{\mu \in \sigma(A)}|\mu|$, suggested in [2] for a class of Blended Implicit Methods (see also [5]). At last, as an example, we list the matrices $A$ and $U$ which define the third order GLM corresponding to the triple $(3,2,2)$,

$$
A=\frac{1}{23}\left(\begin{array}{rr}
22 & -4 \\
36 & 6
\end{array}\right), \quad U=\frac{1}{23}\left(\begin{array}{ll}
-5 & 28 \\
-4 & 27
\end{array}\right),
$$

and those defining the fourth order GLM corresponding to the triple $(4,4,3)$,

$$
A=\left(\begin{array}{rrrr}
0.85795933248329 & -0.22588594615620 & 0.09292560797716 & -0.02384741384935 \\
1.14013555838905 & 0.75295315385401 & -0.30975202659054 & 0.07949137949784 \\
1.16454145194449 & 0.91413597782316 & 0.27338586108581 & -0.07015876367984 \\
1.16304178987375 & 0.90057211551936 & 0.50490826434742 & 0.09507592794253
\end{array}\right),
$$


TABLE 1. Parameters of the blended iteration associated with GLMs, based on GBDF, characterized by the triple $(k, r, \ell)$.

\begin{tabular}{|r|r|r|r|r|r|r|}
\hline$k$ & $r$ & $\ell$ & $\gamma$ & $\tilde{\rho}$ & $\tilde{\rho}_{\infty}$ & $\rho^{*}$ \\
\hline 3 & 2 & 2 & 0.72 & 0.23 & 0.44 & 0.16 \\
4 & 4 & 3 & 0.62 & 0.38 & 0.99 & 0.31 \\
6 & 5 & 4 & 0.61 & 0.57 & 1.56 & 0.47 \\
8 & 6 & 5 & 0.58 & 0.64 & 1.92 & 0.55 \\
10 & 7 & 6 & 0.55 & 0.66 & 2.19 & 0.60 \\
12 & 9 & 7 & 0.53 & 0.73 & 2.64 & 0.70 \\
14 & 10 & 8 & 0.51 & 0.74 & 2.80 & 0.72 \\
16 & 11 & 9 & 0.50 & 0.73 & 2.94 & 0.73 \\
\hline
\end{tabular}

$$
U=\left(\begin{array}{llll}
0.07149661104027 & -0.44184164162566 & 0 & 1.37034503058538 \\
0.09501129653242 & -0.52719452791448 & 0 & 1.43218323138206 \\
0.09704512099537 & -0.53021970356702 & 0 & 1.43317458257165 \\
0.09692014915615 & -0.53024220062923 & 0 & 1.43332205147308
\end{array}\right)
$$

\section{REFERENCES}

1. L. Brugnano. Blended Block BVMs (B 3 VMs): a family of economical implicit methods for ODEs. Jour. Comput. Appl. Mathematics 116 (2000) 41-62.

2. L. Brugnano, C. Magherini. Blended Implementation of Block Implicit Methods for ODEs. Appl. Numer. Math. 42 (2002) 29-45.

3. L. Brugnano, C. Magherini. The BiM code for the numerical solution of ODEs. Jour. Comput. Appl. Mathematics 164-165 (2004) 145-158.

4. L. Brugnano, C. Magherini. Blended Implicit Methods for solving ODE and DAE problems, and their extension for second order problems. Jour. Comput. Appl. Mathematics 205 (2007) 777-790

5. L. Brugnano, C. Magherini. Recent advances in linear analysis of convergence for splittings for solving ODE problems. Appl. Numer. Math. (2008), doi:10.1016/j.apnum.2008.03.008

6. L. Brugnano, C. Magherini, F. Mugnai. Blended Implicit Methods for the numerical solution of DAE problems. Jour. Comput. Appl. Mathematics 189 (2006) 34-50.

7. L. Brugnano, D. Trigiante. Convergence and stability of Boundary Value Methods for ordinary differential equations. Jour. Comput. Appl. Mathematics 66 (1996) 97-109.

8. L.Brugnano, D.Trigiante. Solving Differential Problems by Multistep Initial and Boundary Value Methods, Gordon and Breach Science Publ., 1998.

9. L. Brugnano, D. Trigiante. Block Implicit Methods for ODEs, in Recent Trends in Numerical Analysis, D.Trigiante ed., Nova Science Publ. Inc., New York, 2001, pp. 81-105.

10. K. Burrage, J.C. Butcher. Non-linear stability of a general class of differential equation methods. BIT 20 (1980) 185-203.

11. J.C. Butcher. The Numerical Analysis of Ordinary Differential Equations. Runge-Kutta and General Linear Methods, John Wiley, New York, 1987.

12. J.C. Butcher. Diagonally-implicit multi-stage integration methods. Appl. Numer. Math. 11 (1993) 347-363.

13. J.C. Butcher. General linear methods. Acta Numerica 15 (2006) 157-256.

14. J.C. Butcher, P. Chartier, Z. Jackiewicz. Nordsieck representation of DIMSIMs. Numer. Alg. 16 (1997) 209-230.

15. J.C. Butcher, Z. Jackiewicz. Diagonally implicit general linear methods for ordinary differential equations. BIT 33 (1993) $452-472$.

16. J.C. Butcher, Z. Jackiewicz. Construction of diagonally implicit general linear methods of type 1 and 2 for ordinary differential equations. Appl. Numer. Math. 21 (1996) 385-415.

17. J.C. Butcher, Z. Jackiewicz. Construction of high order diagonally implicit multistage integration methods for ordinary differential equations. Appl. Numer. Math. 27 (1998) 1-12.

18. J.C. Butcher, Z. Jackiewicz. Construction of general linear methods with Runge-Kutta stability properties. Numer. Alg. 36 (2004) 53-72.

19. J.C. Butcher, Z. Jackiewicz, H.D. Mittelmann. Nonlinear optimization approach to the construction of general linear methods of high order. Jour. Comput. Appl. Math. 81 (1997) 181-196.

20. J.C. Butcher, W.M. Wright. The construction of practical general linear methods. BIT 43 (2003) 695-721.

21. H. Podhaisky, R. Weiner, B.A. Schmitt. Linearly-implicit two-step methods and their implementation in Nordsieck form. Appl. Numer. Math. 56 (2006) 374-387. 